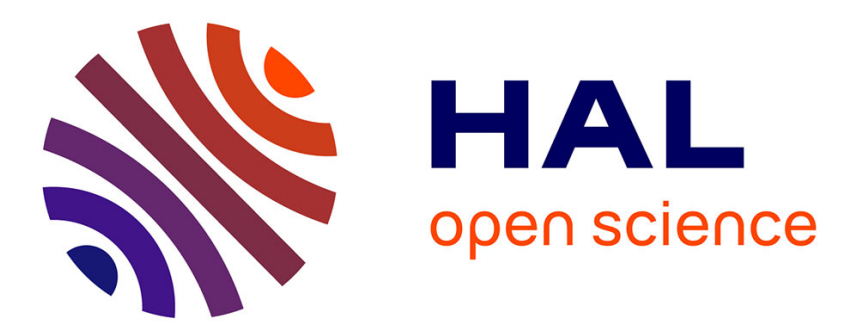

\title{
Set-membership functional diagnosability: Definitions and analysis
}

Carine Jauberthie, Nathalie Verdière, Louise Travé-Massuyès

\section{To cite this version:}

Carine Jauberthie, Nathalie Verdière, Louise Travé-Massuyès. Set-membership functional diagnosability: Definitions and analysis. 2016 Conference on Control and Fault-Tolerant Systems (SysTol), Sep 2016, Barcelona, Spain. 10.1109/SYSTOL.2016.7739794 . hal-01739797

\section{HAL Id: hal-01739797 \\ https://hal.science/hal-01739797}

Submitted on 21 Nov 2018

HAL is a multi-disciplinary open access archive for the deposit and dissemination of scientific research documents, whether they are published or not. The documents may come from teaching and research institutions in France or abroad, or from public or private research centers.
L'archive ouverte pluridisciplinaire HAL, est destinée au dépôt et à la diffusion de documents scientifiques de niveau recherche, publiés ou non, émanant des établissements d'enseignement et de recherche français ou étrangers, des laboratoires publics ou privés. 


\title{
Set-Membership functional diagnosability: definitions and analysis
}

\author{
Carine Jauberthie $^{1}$ and Nathalie Verdière ${ }^{2}$ and Louise Travé-Massuyès ${ }^{1}$
}

\begin{abstract}
This paper introduces the concept of functional diagnosability for nonlinear dynamical uncertain models. Set-membership functional diagnosability is based on the notion of functional signature introduced in [11] extended to the set-membership framework. The link between functional diagnosability and set-membership functional diagnosability is established. Contrary to classical definitions, the study of functional diagnosability highlights some of the residual properties related to the fault acting on the system in time. Set-membership functional diagnosability extends this concept to bounded faults, i.e. faults whose magnitude is unknown but belongs to a bounded set. Numerical simulations for a system based on water tanks illustrate the potential of set-membership functional diagnosability.
\end{abstract}

\section{INTRODUCTION}

Providing models representing physical systems is a common concern spread over all scientific and engineering communities. Complex systems are often subjected to uncertainties that make the modeling task awkward. It is particularly difficult to get an accurate model of the disturbances and noises acting on the system. This may turn the usual stochastic framework inappropriate and this is why stochastic models are sometimes disregarded to the benefit of setmembership (SM) models. SM models naturally cope with uncertainties that are assumed to be bounded but otherwise unknown. This kind of uncertainties can be taken into account by parameters and faults taking set values instead of real values. Hence diagnosis based on SM methods is relevant in many practical situations. Before a system is put into operation and eventually diagnosed, diagnosability analysis is an important stage. Diagnosability is the property that guarantees that the sensored values delivered by the available instrumentation can be processed into an appropriate set of symptoms discriminating different faulty situations. Thus, diagnosability can be checked at design phase so that one knows before hand which faults the diagnoser will be able to discriminate during operation with the specified instrumentation. From another perspective,

\footnotetext{
${ }^{1}$ Carine Jauberthie and Louise Travé-Massuyès are with LAAS CNRS, Université de Toulouse, CNRS, UPS, Toulouse, France, c jaubert, louise@laas.fr

2 Nathalie Verdière is with the University of Le Havre, Normandie Univ, France; ULH, LMAH, F-76600 Le Havre; FR CNRS 3335, 25 rue Philippe Lebon 76600 Le Havre, France, nathalie.verdiere@univ-lehavre.fr
}

this property provides the means to identify the set of - additional - sensors that are required to achieve a given degree of diagnosability.

In this work, a fault is an unpermitted deviation of one parameter of the system from the acceptable standard condition. Hence, faults correspond to parameter variations. In this context, the problem of diagnosability has been shown to be closely related to the problem of univocally inferring the value of the parameters from the measurements. This problem is close to the one of parameter identifiability that ensures that two distinct parameters generate two distinct trajectories for a model. In this work, links between identifiability and diagnosability are recalled through the concept of functional diagnosability introduced in [11].

In most situations, it is not important to distinguish faults by their precise values but one would like to distinguish classes of faults. For example, assessing whether a hole in a pipe has a diameter of $11 \mathrm{~mm}$ or $12 \mathrm{~mm}$ is of no interest. Nevertheless, the order of magnitude is important and one would like to distinguish a $10 \mathrm{~mm}$ diameter hole from a $50 \mathrm{~mm}$ diameter hole. In addition, nominal conditions are generally known with uncertainty i.e. the parameter values are given with some tolerances. We show that this problem can be solved by proposing diagnosability definitions in an SM framework, in particular defining the concepts of strong and weak SM-functional diagnosability.

This paper is organized as follows. In Section II, the core problem is stated and SM-functional diagnosability is presented as an extension of functional diagnosability. The link between functional diagnosability and SM-functional diagnosability is also established. Section III provides an application and numerical results on a water tanks example. The last section concludes the paper and outlines future research directions.

\section{SET-MEMBERSHIP DIAGNOSABILITY}

\section{A. The models}

We are concerned with diagnosability analysis of controlled or uncontrolled SM models of the form described below in which faults and disturbances have 
been explicitly introduced:

$$
\Gamma^{P}\left\{\begin{array}{l}
\dot{x}(t, \theta)=g(x(t, \theta), u(t), f, p), \\
y(t, p)=h(x(t, p), u(t), f, p), \\
x\left(t_{0}, p\right)=x_{0} \in \mathbb{X}_{0}, \\
p \in P \subset \mathscr{U}_{\mathscr{P}}, \\
t_{0} \leq t \leq T,
\end{array}\right.
$$

where $x(t, p) \in \mathbb{R}^{n}$ and $y(t, p) \in \mathbb{R}^{m}$ denote the state variables and the outputs respectively. $u(t)$ is the control vector assumed to account for some random noise considered bounded but otherwise unknown The function $g$ is real and analytic on $M$, where $M$ is an open set of $\mathbb{R}^{n}$ such that $x(t, p) \in M$ for every $t \in\left[t_{0}, T\right]$ and $p$ denotes the parameter vector belonging to a connected set $P \in \mathscr{U}_{\mathscr{P}}$, where $\mathscr{U}_{\mathscr{P}}$ is an open set of $\mathbb{R}^{p}$. The fault vector $f \in \mathbb{R}^{e}$ belongs to a connected set $F \subseteq \mathscr{F}_{S Y S}$, where $\mathscr{F}_{S Y S}$ is the exhaustive set defining the fault domain. $f=0$ means no fault. $Y(P, F, u)$ (resp. $Y(P, F)$ ) denotes the set of outputs, solution of $\Gamma^{P}$ with the input $u$ (resp. when $u=0$ ), the parameter vector $P$ and the fault vector $F$.

In this work, a fault $f_{j}$ is defined as a variation of one parameter and $F_{j} \subseteq \mathscr{F}_{S Y S}$ is a connected set describing a faulty situation characterized by:

-the occurence of $F_{j}$ introduces disturbances that are assumed to be bounded,

-the magnitude of the fault is assumed to belong to a bounded set.

$F_{j}$ is called a "bounded fault".

Using elimination theory, some differential polynomials or analytical redundancy relations (ARR) linking system inputs, outputs and their derivatives can be obtained. In the last decade, algorithms for obtaining such ARRs have been developed and implemented in softwares like Maple [1]. They are based on differential algebra [7] and consist in eliminating unobservable state variables in order to obtain the relations. The use of ARRs makes possible to detect [6], isolate, and estimate the severity of a fault acting on the system.

Several definitions of diagnosability have been formed around the use of ARRs. The classical diagnosability definition consists in comparing fault signatures [2]. The fault signature is a function which associates to a fault the set of indicators, also called residuals, obtained from the ARRs fed by measured variables. Typically, the fault signature of $f_{j}$ is the $m$ vector formed of zeros and ones: when the fault $f_{j}$ acts on the residual $\rho_{i}$ formed from an ARR, the number 1 is put in the $i^{t h}$ component of the $f_{j}$ 's signature otherwise it is 0 . The authors of [3] consider that a system is diagnosable if each fault can be written as the solution of a polynomial equation in $f_{j}$ and a finite number of time derivatives of inputs and outputs. This definition is close to the one of identifiability proposed by [10]. However, the definition of diagnosability proposed in [3] is based on the obtention of particular differential polynomials which may require a lot of manipulations of the model equations. In case of complex models, it is often impossible to obtain such polynomials even using dedicated symbolic softwares. Furthermore, the order of derivatives is so high that they are hardly suitable for fault detection.

In [11], the authors propose to relax the constraints of [3] and to use ARRs involving one or more faults for studying diagnosability and detecting faults, the advantage being to easier the obtention of ARRs with derivatives of lower order. The obtention of such ARRs is based on the work of [4]. From these particular ARRs, the definition of functional diagnosability was proposed in [11]. The authors showed that if the model is identifiable with respect to the faults, then the residuals of these ARRs forming the functional signature have distinct trajectories. Furthermore, single faults can be easily detected.

As explained in the introduction, one may be interested in distinguishing only classes of faults, hence the notion of $S M$ diagnosability that we introduce in this paper. SM diagnosability comes through the notion of functional signature which is extended to the stochastic context.

In the following subsections, the obtention through variable elimination of ARRs expressions is presented in the stochastic framework.

\section{B. Obtention of ARRs}

In [11], the authors propose to use specific ARRs for defining functional diagnosability and achieving fault detection in algebraic dynamic systems. These ARRs are obtained from the Rosenfeld-Groebner algorithm implemented in Maple [1]. An ARR is a relation deduced from the model of the system that links the system inputs and outputs and their derivatives. Provided that derivatives can be estimated, an ARR is hence a testable relation in the sense that it can be evaluated with the measurements and this is why it is useful for fault detection and isolation. For any vector $\vartheta$, let us define $\bar{\vartheta}$ to stand for $\vartheta$ and its time derivatives up to some (unspecified) order. Then ARRs can be put in the following form:

$$
\begin{aligned}
w_{i}(\bar{y}, \bar{u}, f, p)= & m_{0, i}(\bar{y}, \bar{u}, p)-\sum_{k=1}^{n_{i}} \gamma_{k}^{i}(f, p) m_{k, i}(\bar{y}, \bar{u}) \\
& =w_{0, i}(\bar{y}, \bar{u}, p)-w_{1, i}(\bar{y}, \bar{u}, f, p)
\end{aligned}
$$

where $\left(\gamma_{k}^{i}(f, p)\right)_{1 \leq k \leq n_{i}}$ are rational in $f$ and $p, \gamma_{v}^{i} \neq \gamma_{w}^{i}$ $(v \neq w)$ and $\left(m_{k, i}(y, u)\right)_{1 \leq k \leq n_{i}}$ are differential polynomials with respect to $\bar{y}$ and $\bar{u} . w_{0, i}(\bar{y}, \bar{u}, p)$ is equal to $m_{0, i}(\bar{y}, \bar{u}, p)$, hence the first part of the polynomial is not identically equal to zero and does not contain components of $f$. It corresponds to the residual computation form whereas $w_{1, i}(\bar{y}, \bar{u}, f, p)$ is known as the residual internal form. The polynomials $w_{i}$ are called input-output polynomials.

We can notice that in the classical diagnosability 
framework, if two faults act on the same residuals, their fault signatures are the same. Thus, theoretically, it is not possible to distinguish them. With functional diagnosability, the residuals can have different behaviors depending on how the faults are involved in their expressions.

\section{Functional diagnosability}

The functional signature of a fault $f_{j}$ is given by the vector formed by the internal form of the $m$ residuals by considering that only the fault $f_{j}$ is acting on the system. In other words, it is given by the vector whose $i$ th component is $w_{1, i}\left(\bar{y}, \bar{u}, f_{j}, p\right)$, that is the polynomial obtained from $w_{1, i}(\bar{y}, \bar{u}, f, p)$ by considering all the components of $f$ equal to zero but the $j$ th component equal to $f_{j}$. The following definition of functional fault signature proposed in [11] is recalled.

Definition 2.1: The functional fault signature is a function $F$ Sig which associates to a fault $f_{j}$, the vector $\left(w_{1, i}\left(\bar{y}, \bar{u}, f_{j}, p\right)\right)_{i=1, \ldots, m}$.

We denote by $F \operatorname{Sig}^{(i)}\left(f_{j}\right)=w_{1, i}\left(\bar{y}, \bar{u}, f_{j}, p\right)$ the ith component of $F \operatorname{Sig}\left(f_{j}\right)$.

$F \operatorname{Sig}\left(f_{j}\right)$ is a vector of functions whose components constitute each a trajectory. The following definitions propose to link the functional signature and the notions of discriminality and diagnosability. The first one is true for all inputs, whereas the second one is verified only for one input.

Definition 2.2: Two different faults $f_{j}$ and $f_{l}$ are input-strongly functionally discriminable if $F \operatorname{Sig}\left(f_{j}\right) \neq F \operatorname{Sig}\left(f_{l}\right)$ in the sense that for all input $u$, there exists at least one index $i^{*}$ and a time interval $\left[t_{1}, t_{2}\right]$ such that for all $t \in\left[t_{1}, t_{2}\right]$, $\operatorname{FSig}^{\left(i^{*}\right)}\left(f_{j}\right) \neq \operatorname{FSig}^{\left(i^{*}\right)}\left(f_{l}\right)$.

When all the faults are input-strongly functionally discriminable, the model is said input-strongly functionally diagnosable.

Definition 2.3: Two different faults $f_{j}$ and $f_{l}$ are input-weakly functionally discriminable if $F \operatorname{Sig}\left(f_{j}\right) \neq$ $F \operatorname{Sig}\left(f_{l}\right)$ in the sense that there exits at least one input $u$ for which there exists at least one index $i^{*}$ and a time interval $\left[t_{1}, t_{2}\right]$ such that for all $t \in\left[t_{1}, t_{2}\right]$, $\operatorname{FSig}^{\left(i^{*}\right)}\left(f_{j}\right) \neq \operatorname{FSig}^{\left(i^{*}\right)}\left(f_{l}\right)$.

When all the faults are input-weakly functionally discriminable, the model is said input-weakly functionally diagnosable.

When the model is uncontrolled, we have the following definition:

Definition 2.4: Two different faults $f_{j}$ and $f_{l}$ are functionally discriminable if $F \operatorname{Sig}\left(f_{j}\right) \neq F \operatorname{Sig}\left(f_{l}\right)$ in the sense that there exists at least one index $i^{*}$, a time interval $\left[t_{1}, t_{2}\right]$ such that for all $t \in\left[t_{1}, t_{2}\right]$, $\operatorname{FSig}^{\left(i^{*}\right)}\left(f_{j}\right) \neq$ Fig $^{\left(i^{*}\right)}\left(f_{l}\right)$.

When all the faults are functionally discriminable, the model is said functionally diagnosable.
Remark- Signatures of faults can be collected in a table for which the component intersecting the $i^{\text {th }}$ line and $j^{\text {th }}$ column contains the $i^{t h}$ residual for the fault $f_{j}$, that is $w_{1, i}\left(\bar{y}, \bar{u}, f_{j}, p\right)$. An example is proposed below:

Example 2.1: We consider the following model:

$$
\left\{\begin{array}{l}
\dot{x}_{1}=\left(p_{1}+f_{1}\right)\left(p_{2}+f_{2}\right) x_{1}^{2}+x_{1} x_{2}, \\
\dot{x}_{2}=\left(p_{2}+f_{2}\right)\left(p_{3}+f_{3}\right) x_{2}^{2}+x_{2} x_{3}, \\
\dot{x}_{3}=\left(p_{1}+f_{1}\right)\left(p_{3}+f_{3}\right) x_{3}^{2}+x_{1} x_{3}, \\
y_{1}=x_{1}, y_{2}=x_{2}, y_{3}=x_{3} .
\end{array}\right.
$$

It is easy to verify that:

$$
\begin{gathered}
w_{0,1}(\bar{y}, \bar{u}, p)=\dot{y}_{1}-y_{1} y_{2}-p_{1} p_{2} y_{1}^{2}, \\
w_{0,2}(\bar{y}, \bar{u}, p)=\dot{y}_{2}-y_{2} y_{3}-p_{2} p_{3} y_{2}^{2}, \\
w_{0,3}(\bar{y}, \bar{u}, p)=\dot{y}_{3}-y_{1} y_{3}-p_{1} p_{3} y_{3}^{2} . \\
w_{1,1}(\bar{y}, \bar{u}, f, p)=\left(p_{1} f_{2}+p_{2} f_{1}+f_{1} f_{2}\right) y_{1}^{2}, \\
w_{1,2}(\bar{y}, \bar{u}, f, p)=\left(p_{2} f_{3}+p_{3} f_{2}+f_{2} f_{3}\right) y_{2}^{2}, \\
w_{1,3}(\bar{y}, \bar{u}, f, p)=\left(p_{1} f_{3}+p_{3} f_{1}+f_{1} f_{3}\right) y_{3}^{2} .
\end{gathered}
$$

The functional signatures of the faults $f_{1}, f_{2}$ and $f_{3}$ are given by:

$\operatorname{Fig}\left(f_{1}\right)=\left(p_{2} f_{1} y_{1}^{2}, 0, p_{3} f_{1} y_{3}^{2}\right)^{T}$,

$F \operatorname{Sig}\left(f_{2}\right)=\left(p_{1} f_{2} y_{1}^{2}, p_{3} f_{2} y_{2}^{2}, 0\right)^{T}$,

$F \operatorname{Sig}\left(f_{3}\right)=\left(0, p_{2} f_{3} y_{2}^{2}, p_{1} f_{3} y_{3}^{2}\right)^{T}$.

Functional diagnosability can be analysed with the following table:

\begin{tabular}{l|ccc}
$F \operatorname{Sig}(f) / \mathrm{f}$ & $f_{1}$ & $f_{2}$ & $f_{3}$ \\
\hline$F \operatorname{Sig}^{(1)}(f)$ & $p_{2} f_{1} y_{1}^{2}$ & $p_{1} f_{2} y_{1}^{2}$ & 0 \\
$F \operatorname{Sig}^{(2)}(f)$ & 0 & $p_{3} f_{2} y_{2}^{2}$ & $p_{2} f_{3} y_{2}^{2}$ \\
$F_{\operatorname{Sig}^{(3)}}(f)$ & $p_{3} f_{1} y_{3}^{2}$ & 0 & $p_{1} f_{3} y_{3}^{2}$
\end{tabular}

Clearly, for $j, l=1,2,3, j \neq l, F \operatorname{Sig}\left(f_{j}\right) \neq F \operatorname{Sig}\left(f_{l}\right)$ and the model is functionally diagnosable.

Let us notice that the functional signatures are of the form:

$$
F \operatorname{Sig}\left(f_{i}\right)=\left(\sum_{k=1}^{n_{i}} \gamma_{k}^{i}\left(f_{i}, p\right) m_{k, i}(\bar{y}, \bar{u})\right)_{i=1, \ldots, m} .
$$

\section{Extension to the set-membership framework}

Considering a vector of faults $F \subseteq \mathscr{F}_{S Y S}$, let us denote by $F_{j}$ the vector derived from $F$ by setting all the components equal to zero except the jth component. Notice that $F_{j}$ is a bounded fault given by a connected set.

Definition 2.5: The SM-functional signature is a function $F_{S i g}$ which associates to the connected set $F_{j}$ the interval vector $\left(w_{1, i}\left(\bar{Y}(P, u), \bar{u}, F_{j}, P\right)\right)_{i=1, \ldots, m}$. $F \operatorname{Sig}_{S M}^{(i)}\left(F_{j}\right)$ denotes the ith component of $F \operatorname{Sig}_{S M}\left(F_{j}\right)$ and corresponds to an interval function. $F_{S i g}^{(i)}\left(F_{j}\right)$ consists of a set of trajectories generated in the presence of $F_{j}$ and can be viewed as a tube of trajectories on the time interval $\left[t_{0}, T\right]$.

The aim is now to give definitions for distinguishing the set of trajectories generated by $F_{1}$ and $F_{2}$ and more precisely for distinguishing the two tubes of trajectories generated by these bounded faults. The first 
definition refers to weak SM-functional diagnosability and permits an intersection of the two tubes on a time interval. The second refers to strong SM-functional diagnosability and requires the two tubes .

Definition 2.6: Two bounded faults $F_{1}$ and $F_{2}$ are weakly SM-functionally discriminable if $F \operatorname{Sig}_{S M}\left(F_{1}\right)$ and $F_{\operatorname{Sig}_{S M}}\left(F_{2}\right)$ are distinct in the sense that there exists at least one index $i^{*}$ and a time interval $\left[t_{1}, t_{2}\right]$ such that for all $t \in\left[t_{1}, t_{2}\right], F \operatorname{Sig}_{S M}^{\left(i^{*}\right)}\left(F_{1}\right) \nsubseteq F \operatorname{Sig}_{S M}^{\left(i^{*}\right)}\left(F_{2}\right)$ or $F_{S i g}^{\left(i^{*}\right)}\left(F_{2}\right) \nsubseteq F \operatorname{Fig}_{S M}^{\left(i^{*}\right)}\left(F_{1}\right)$ and $F_{S i g}^{(i *)}\left(F_{1}\right) \cap$ $F_{S i g}^{(i *)}\left(F_{2}\right) \neq \emptyset$.

Definition 2.7: Two bounded faults $F_{1}$ and $F_{2}$ are strongly SM-functionally discriminable if there exists an index $i^{*} \in\{1, \ldots, m\}$ and a time interval $\left[t_{1}, t_{2}\right]$ such that for all $t \in\left[t_{1}, t_{2}\right], F \operatorname{Sig}_{S M}^{\left(i^{*}\right)}\left(F_{1}\right) \cap F \operatorname{Sig}_{S M}^{\left(i^{*}\right)}\left(F_{2}\right)=\emptyset$.

Definition 2.8: The model $\Gamma^{P}$ given by (1) is weakly SM-functionally diagnosable for $\mathscr{F}_{S Y S}$ if any two bounded faults $F_{1}, F_{2} \subseteq \mathscr{F}_{S Y S}$ are weakly SMfunctionally discriminable.

Definition 2.9: The model $\Gamma^{P}$ given by (1) is strongly SM-functionally diagnosable for $\mathscr{F}_{S Y S}$ if all pair $F_{1}, F_{2} \subseteq \mathscr{F}_{S Y S}$ of faults are strongly SMfunctionally discriminable.

The above definitions are given for uncontrolled systems and "weak" and "strong" refer to properties of the tube of trajectories. If the system is controlled and the input $u(t)$ is not equal to zero then the definitions are also available in their input-weak and input-strong form simarly to definitions 2.3 and 2.3.

\section{E. Links between functional diagnosability and SM- functional diagnosability}

The following proposition shows that strong SMfunctional diagnosability can be inferred from functional diagnosability.

Proposition 2.1: In the case of single faults, if the model $\Gamma^{P}$ is functionally diagnosable for any $p \in P$ then the model is strongly SM-functionally diagnosable.

Proof - For two distinct faults $f_{j} \in F_{j}$ and $f_{l} \in F_{l}$ and for any parameter $p \in P$, by assumption, the trajectories generated by $F \operatorname{Sig}\left(f_{j}\right)$ and $F \operatorname{Sig}\left(f_{l}\right)$ are distinct on an interval $\left[t_{1}, t_{2}\right]$, thus the two tubes of trajectories $F \operatorname{Sig}_{S M}\left(F_{j}\right)$ and $F \operatorname{Sig}_{S M}\left(F_{l}\right)$ have an empty intersection on $\left[t_{1}, t_{2}\right]$.

F. Link between identifiability, functional diagnosability and SM-functional diagnosability

In [11], a link has been established between functional diagnosability and identifiability. Identifiability establishes an unambiguous mapping between the parameters and the output trajectories. The following proposition gives a necessary and sufficient condition to verify identifiability of a model.

Proposition 2.1: Assume that there exists an index set $I_{0} \subseteq\{1, \ldots, m\}$ such that for all $i \in$
$I_{0}$, the functional determinants $\triangle w_{1, i}(\bar{y}, \bar{u}, f, p)=$ $\operatorname{det}\left(m_{k, i}(\bar{y}, \bar{u}, f, p), k=1, \ldots, n_{i}\right)$ are not identically equal to zero ${ }^{1}$. The model (1) is globally identifiable with respect to the faults if and only if the function $\phi$ defined by :

$\phi: f=\left(f_{1}, \ldots, f_{p}\right) \mapsto\left(\gamma_{1}^{i}(f, p), \ldots, \gamma_{n_{i}}^{i}(f, p)\right)_{i \in I_{0}}$ is injective.

The following proposition gives the link between functional diagnosability and identifiability of the model with respect to the faults.

Proposition 2.2: Assume that there exists an index set $I_{0} \subseteq\{1, \ldots, m\}$ such that for all $i \in$ $I_{0}$, the functional determinants $\triangle w_{1, i}(\bar{y}, \bar{u}, f, p)=$ $\operatorname{det}\left(m_{k, i}(\bar{y}, \bar{u}, f, p), k=1, \ldots, n_{i}\right)$ are not identically equal to zero. If the model is globally identifiable with respect to the faults then the model is (input-strongly) functionally diagnosable. The reciprocal is not true.

Thus, using Proposition 2.2 and Proposition 2.1, it comes that for verifying strong SM-functional diagnosability, it is sufficient to verify that:

1) the functional determinants $\triangle w_{1, i}(\bar{y}, \bar{u}, f, p)=$ $\operatorname{det}\left(m_{k, i}(\bar{y}, \bar{u}, f, p), k=1, \ldots, n_{i}\right)$ are not identically equal to zero for a set of indexes $I_{0}$

2) the function $\phi$ is injective.

\section{EXAMPLE: WATER-TANKS}

Consider the two coupled water tanks modeled by:

$$
\left\{\begin{array}{l}
\dot{x}_{1}(t, p)=a_{1} u(t)-a_{2} \sqrt{x_{1}(t, p)}, \\
\dot{x}_{2}(t, p)=a_{3} \sqrt{x_{1}(t, p)}-a_{4} \sqrt{x_{2}(t, p)}, \\
y_{1}(t, p)=a_{5} \sqrt{x_{1}(t, p)} \\
y_{2}(t, p)=a_{6} \sqrt{x_{2}(t, p)},
\end{array}\right.
$$

where $p=\left(a_{i}\right)_{i=1, \ldots, 6}, a_{i} \neq 0$, is the parameter vector, $x=\left(x_{1}, x_{2}\right)^{T}$ represents the state vector and corresponds to the level in each tank, and $u \neq \equiv$ is the input vector. The water level in the tanks can vary between 0 and 10.

Let $f_{1}$ denote an unknown additive fault on the actuator signal, $f_{2}$ and $f_{3}$ are additive faults refering to the two sensors on the output of each of the water tanks, and $f_{4}$ is a clogging fault. We assume that the faults are bounded such that $f_{i} \in[0,1], \mathrm{i}=1, \ldots, 4$. $f_{4}=1$ represents a fully clogged pipe and $0<f_{4}<1$ represents partial clogging. In the faulty scenarios, the faults are introduced at time $t=20 \mathrm{~s}$.

\footnotetext{
${ }^{1}$ This assumption consists in verifying the linear independence of the $m_{k, i}(\bar{y}, \bar{u}, f, p), k=1, \ldots, n_{i}$, by checking that the functional determinant given by the Wronskian [5]
}

$\Delta w_{1, i}(\bar{y}, \bar{u}, f, p)=\left|\left(\begin{array}{ccc}m_{1, i}(\bar{y}, \bar{u}, f, p) & \ldots & m_{n_{i}, i}(\bar{y}, \bar{u}, f, p) \\ m_{1, i}(\bar{y}, \bar{u}, f, p)^{(1)} & \ldots & m_{n_{i}, i}(\bar{y}, \bar{u})^{(1)} \\ & \ddots & \\ m_{1, i}(\bar{y}, \bar{u}, f, p)^{\left(n_{i}-1\right)} & \ldots & m_{n_{i}, i}(\bar{y}, \bar{u}, f, p)^{\left(n_{i}-1\right)}\end{array}\right)\right|$

is not identically equal to zero. If there exists a time point at which the Wronskian is non-zero, then the monomials are linearly independent. 
In order to use the Rosenfeld-Groebner algorithm implemented in Maple 16, auxiliary variables $z_{1}(t, p)=\sqrt{x_{1}(t, p)}$ and $z_{2}(t, p)=\sqrt{x_{2}(t, p)}$ are introduced and the model, including the representation of the four faults, is rewritten as:

$$
\left\{\begin{array}{l}
\dot{x}_{1}(t, p)=a_{1}\left(u(t)+f_{1}\right)-a_{2}\left(1-f_{4}\right) z_{1}(t, p) \\
\dot{x}_{2}(t, p)=a_{3}\left(1-f_{4}\right) z_{1}(t)-a_{4} z_{2}(t, p) \\
z_{1}(t, p)^{2}=x_{1}(t, p) \\
z_{2}(t, p)^{2}=x_{2}(t, p) \\
y_{1}(t, p)=a_{5}\left(1-f_{4}\right) z_{1}(t, p)+f_{2} \\
y_{2}(t, p)=a_{6} z_{2}(t, p)+f_{3} \\
\dot{f}_{i}=0
\end{array}\right.
$$

According to the Rosenfeld-Groebner algorithm, the two ARRs are:

$$
\begin{aligned}
& w_{1}(\bar{y}, \bar{u}, f, p)=w_{0,1}(\bar{y}, \bar{u}, p)-w_{1,1}(\bar{y}, \bar{u}, f, p) \\
& w_{2}(\bar{y}, \bar{u}, f, p)=w_{0,2}(\bar{y}, \bar{u}, p)-w_{1,2}(\bar{y}, \bar{u}, f, p)
\end{aligned}
$$

where:

$$
\begin{aligned}
& w_{0,1}=-u a_{1} a_{5}^{2}+\left(a_{2} a_{5}+2 \dot{y}_{1}\right) y_{1}, \\
& w_{1,1}=2 \dot{y}_{1} f_{2}-\left(f_{4}^{2} a_{2} a_{5}-2 f_{4} a_{2} a_{5}\right) y_{1} \\
& -\left(-f_{4}^{2} a_{1} a_{5}^{2}+2 f_{4} a_{1} a_{5}^{2}\right) u \\
& +f_{4}^{2} f_{2} a_{2} a_{5}+f_{4}^{2} f_{1} a_{1} a_{5}^{2}-2 f_{4} f_{2} a_{2} a_{5} \\
& -2 f_{4} f_{1} a_{1} a_{5}^{2}+f_{2} a_{2} a_{5}+f_{1} a_{1} a_{5}^{2}, \\
& w_{0,2}=2 a_{5} \dot{y}_{2} y_{2}-a_{3} a_{6}^{2} y_{1}+a_{4} a_{5} a_{6} y_{2}, \\
& w_{1,2}=2 \dot{y}_{2} f_{3} a_{5}+f_{3} a_{4} a_{5} a_{6}-f_{2} a_{3} a_{6}^{2} .
\end{aligned}
$$

Hence,

$$
\begin{aligned}
F \operatorname{Sig}\left(f_{1}\right) & =\left(-f_{1} a_{1} a_{5}^{2}, 0\right)^{T} \\
F \operatorname{Sig}\left(f_{2}\right) & =\left(-2 \dot{y}_{1} f_{2}-f_{2} a_{2} a_{5}, f_{2} a_{3} a_{6}^{2}\right)^{T} \\
F \operatorname{Sig}\left(f_{3}\right)= & \left(0,-2 \dot{y}_{2} f_{3} a_{5}-f_{3} a_{4} a_{5} a_{6}\right)^{T} \\
F \operatorname{Sig}\left(f_{4}\right)= & \left(y_{1} f_{4}^{2} a_{2} a_{5}-2 y_{1} f_{4} a_{2} a_{5}\right. \\
& \left.\quad-u f_{4}^{2} a_{1} a_{5}^{2}+2 u f_{4} a_{1} a_{5}^{2}, 0\right)^{T} .
\end{aligned}
$$

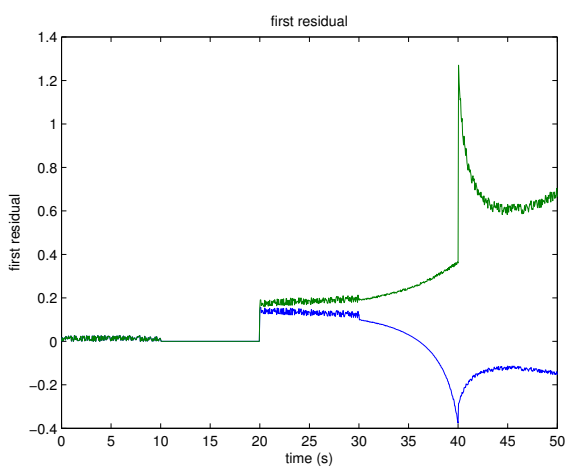

Fig. 1. Residual $\rho_{1}$ when fault $f_{1}$ is introduced at $t=20 \mathrm{~s}$

The functional determinants $\Delta w_{1,1}(\bar{y}, \bar{u})=$ $\operatorname{det}\left(1, u, y_{1}, \dot{y}_{1}\right)=\dot{u}\left(\ddot{y}_{1} y_{1}^{(4)}-y_{1}^{(3)^{2}}\right)-\ddot{u}\left(\dot{y}_{1} y_{1}^{(4)}-\right.$ $\left.y_{1}^{(3)} \ddot{y}_{1}\right)+u^{(3)}\left(\dot{y}_{1} y_{1}^{(3)}-\ddot{y}_{1}^{2}\right)$ and $\Delta w_{1,2}(y, u)=$ $\operatorname{det}\left(\dot{y}_{2}, 1\right)=-\ddot{y}_{2}$ are not identically equal to zero if $u$

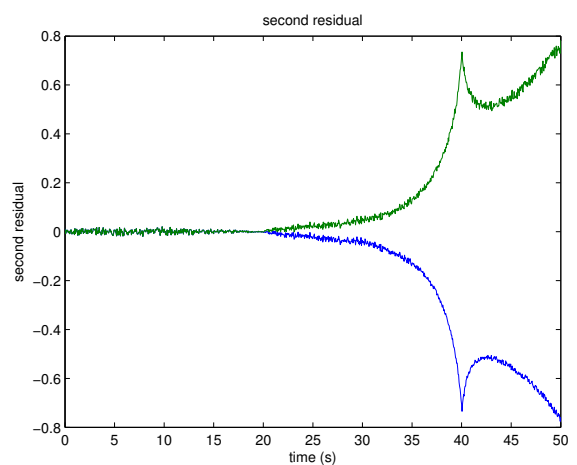

Fig. 2. Residual $\rho_{2}$ when fault $f_{1}$ is introduced at $t=20 \mathrm{~s}$

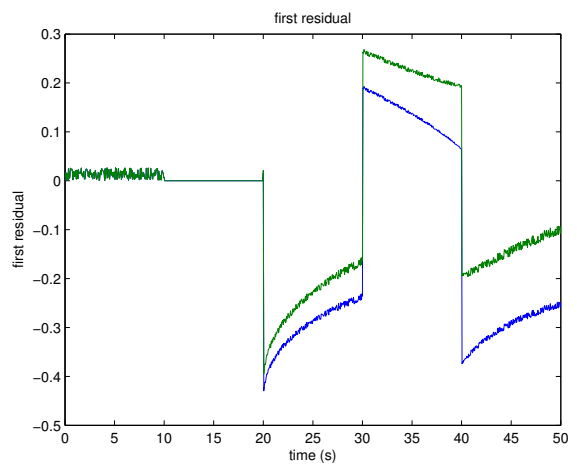

Fig. 3. Residual $\rho_{1}$ when fault $f_{4}$ is introduced at $t=20 \mathrm{~s}$

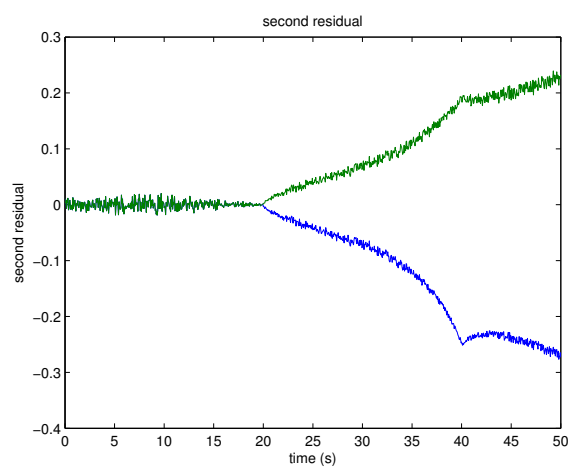

Fig. 4. Residual $\rho_{2}$ when fault $f_{4}$ is introduced at $t=20 \mathrm{~s}$

is not identically equal to a constant. The function

$$
\begin{aligned}
\phi(f)= & \left(-2 f_{2}, f_{4}^{2} a_{2} a_{5}-2 f_{4} a_{2} a_{5},\right. \\
& -f_{4}^{2} a_{1} a_{5}^{2}+2 f_{4} a_{1} a_{5}^{2}, \\
& -f_{4}^{2} f_{2} a_{2} a_{5}-f_{4}^{2} f_{1} a_{1} a_{5}^{2}+2 f_{4} f_{2} a_{2} a_{5}+ \\
& 2 f_{4} f_{1} a_{1} a_{5}^{2}-f_{2} a_{2} a_{5}-f_{1} a_{1} a_{5}^{2}, \\
& \left.-2 f_{3} a_{5},-f_{3} a_{4} a_{5} a_{6}+f_{2} a_{3} a_{6}^{2}\right)
\end{aligned}
$$

is clearly injective for $\left.f_{4} \in\right] 0,1[$. Thus the model is globally identifiable at $f$ with respect to $] 0,1\left[{ }^{4}\right.$. According to Proposition 2.2, the model is hence strongly SM-functionally diagnosable. Interestingly, we can notice that this model is not diagnosable in 
the classical sense.

In the simulations, a simple controller is used to control the water level in the upper tank to follow a square reference signal. The two sensors are disturbed by a truncated Gaussian noise so that the relative error has a maximal value of 0.1 . The parameters of the model are equal to $a_{1}=a_{2}=a_{3}=a_{4}=0.3$, $a_{5}=a_{6}=1$.

The residuals in their computation form $\rho_{1}=$ $-u a_{1} a_{5}^{2}+\left(a_{1} a_{5}+2 \dot{y}_{1}\right) y_{1}$ and $\rho_{2}=2 a_{5} \dot{y}_{2} y_{2}-a_{3} a_{6}^{2} y_{1}+$ $a_{4} a_{5} a_{6} y_{2}$, deduced from (9), are used for detecting the faults. The initial conditions $x_{1}(0), x_{2}(0)$ are assumed to belong to the bounded intervals $[0.55,0.65]$ and $[0.9,1.1]$ respectively. We present the two scenarios in which the faults $f_{1}$ and $f_{4}$ are introduced at time $t=$ $20 s$, respectively. $f_{1}$ and $f_{4}$ are assumed to belong to the interval $[0.45,0.55]$. The derivatives are estimated using an HOSM differentiator ([8], [9]). Figures 1 4 represent the residuals $\rho_{1}$ and $\rho_{2}$ when these two faults act on the system, the blue line corresponds to the lower bound of the residuals and the green line to the upper bound.

Comparing the residuals $\rho_{1}$ in Figures 1 and 3, we can notice that the two tubes of trajectories generated by the faults $f_{1}$ and $f_{4}$ have an empty intersection on the interval $\left[t_{1}, t_{2}\right]=[20,30]$ and this illustrates the fact that the model is strongly SM-functionally diagnosable.

\section{CONCLUSION}

In this paper, a complete procedure to test the new concept of SM-functional diagnosability is proposed. This test is based on the links between identifiability, functional diagnosability and SM-functional diagnosability. A numerical example based on water-tanks is used to illustrate the approach and confirm the relevancy of the new concept. Future work will consider the link between SM-identifiability proposed in [6] and SM-functional diagnosability.

\section{REFERENCES}

[1] F. Boulier, D. Lazard, F. Ollivier, and M. Petitot. Computing representation for radicals of finitely generated differential ideals. Technical report, IT-306, Université Lille I, LIFL, 59655, Villeneuve d'Ascq, 1997.

[2] M.O. Cordier, L. TravÃl'-MassuyÃÍls, and X. Pucel. Comparing diagnosability in continuous and discrete-event systems. In In Proceedings of the 17th International Workshop on Principles of Diagnosis, DX'06, pages 55-60, 2006.

[3] J.C. Cruz-Victoria, R. Martinez-Guerra, and JJ. RinconPasaye. On linear systems diagnosis using differential and algebraic methods. Journal of the Franklin Institute, 345:102118, 2008.

[4] L. Denis-Vidal, G. Joly-Blanchard, and C. Noiret. Some effective approaches to check identifiability of uncontrolled nonlinear systems. Mathematics and Computers in Simulation, 57:35-44, 2001.

[5] L. Denis-Vidal, G. Joly-Blanchard, and C. Noiret. Some effective approaches to check identifiability of uncontrolled nonlinear systems. Mathematics and Computers in Simulation, 57:35-44, 2001.
[6] C. Jauberthie, N. Verdière, and L. Travé-Massuyès. Fault detection and identification relying on set-membership identifiability. Annual Reviews in Control, 37:129-136, 2013.

[7] E.R. Kolchin. Differential algebra and algebraic groups. Academic Press, New York, 1973.

[8] A. Levant. Robust exact differentiation via sliding mode technique. Automatica, 34:379-384, 1998.

[9] A. Levant. Higher-order sliding modes, differentiation and output-feedback control. Int J. Control, 76:924-941, 2003.

[10] L. Ljung and T. Glad. On global identifiability for arbitrary model parametrizations. Automatica, 30:265-276, 1994.

[11] N. Verdière, C. Jauberthie, and L. Travé-Massuyès. Functional diagnosability and detectability of nonlinear models based on analytical redundancy relations. Journal of Process Control, 35:1-10, 2015. 